\title{
Germanica
}

$47 \mid 2010$

« Krack ! Tschock ! Pflatsch ! Bummmm ! La BD de langue allemande (à suivre...) »

\section{Nick Knatterton et le « rêve allemand » des années cinquante}

Nick Knatterton und «der deutsche Travun» der 50er Jahre

Nick Knatterton and the «German dream» of the Fifties

Ingeborg Rabenstein-Michel

\section{OpenEdition}

Journals

Édition électronique

URL : https://journals.openedition.org/germanica/1103

DOI : 10.4000/germanica. 1103

ISSN : 2107-0784

Éditeur

Université de Lille

\section{Édition imprimée}

Date de publication : 31 décembre 2010

Pagination : 51-66

ISBN : 9782913857261

ISSN : 0984-2632

Référence électronique

Ingeborg Rabenstein-Michel, « Nick Knatterton et le « rêve allemand » des années cinquante »,

Germanica [En ligne], 47 | 2010, mis en ligne le 01 décembre 2012, consulté le 21 septembre 2021.

URL : http://journals.openedition.org/germanica/1103; DOI : https://doi.org/10.4000/germanica.1103

Ce document a été généré automatiquement le 21 septembre 2021.

(c) Tous droits réservés 


\section{Nick Knatterton et le « rêve allemand » des années cinquante}

Nick Knatterton und «der deutsche Travun» der 50er Jahre

Nick Knatterton and the «German dream» of the Fifties

Ingeborg Rabenstein-Michel

Nick Knatterton est sans doute la BD la plus emblématique d'une Allemagne de l'aprèsguerre qu'elle accompagne et commente à un moment charnière de son histoire. Premier véritable classique de la BD allemande ${ }^{1}$, parodie réjouissante du genre et satire de la société, elle a connu un énorme succès. Aujourd'hui, elle peut être considérée comme un document particulièrement intéressant sur les années cinquante, la reconstruction et le miracle économique naissant. 


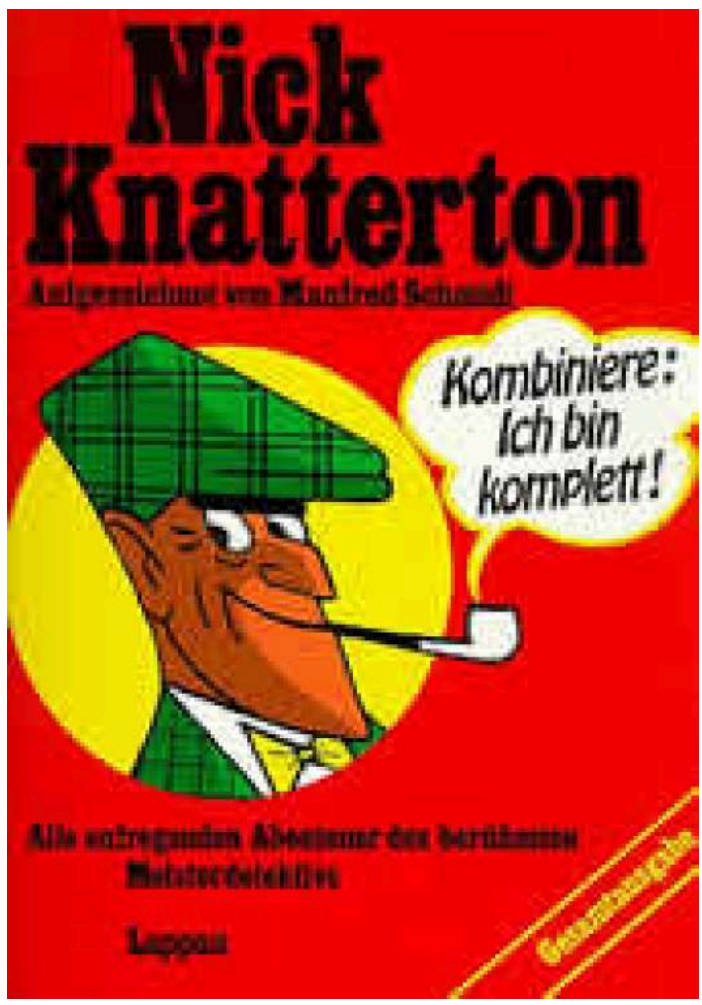

Page de couverture des aventures de Nick Knatterton publiées par les Éditions Lappan

\section{Le coup de maître de Schmidt}

2 À partir de 1945, les Allemands découvrent, sur fond de champs de ruines et grâce à l'Occupation, les premiers éléments d'un nouveau mode de vie, cet «american way of life » qui deviendra un modèle de société : dans ce pays resté coupé du monde depuis l'avènement du national-socialisme, dévasté par la guerre et confronté au poids de sa responsabilité voire culpabilité, les G.I.s importent chewing gum, bas nylon, swing - et les derniers comic-books, plébiscités par la jeunesse. Le régime hitlérien avait considéré la bande dessinée comme « débilitante » et fondamentalement incompatible avec ses propres «valeurs $»^{2}$. L'Allemagne de l'après-guerre reconduit cette méfiance envers le genre ${ }^{3}$, tout comme les États-Unis à cette même époque ${ }^{4}$. La BD est accusée de séduire la jeunesse, de la mener tout droit à la décadence culturelle voire à la délinquance juvénile ${ }^{5}$. Peu de ces comics sont d'ailleurs destinés aux adultes qui s'intéressent plutôt à une autre spécialité venue des US, le "Reader's Digest » dont la première édition en langue allemande paraît en 1948. Cette publication/compilation bien-pensante - tout l'opposé des BD donc - leur semblait un vecteur culturel autrement plus acceptable.

3 C'est dans ce contexte que la revue Quick - fondée en 1948 et longtemps parmi les magazines à plus fort tirage du pays - publie, en 1950, la première aventure de Nick Knatterton. Le succès sera aussi inattendu que complet, et durera une décennie. D'après son créateur, il avait pourtant reposé sur un malentendu. Considérant les bandes dessinées américaines comme une sorte de degré zéro de la culture, Manfred Schmidt (1913-1999) affirme avoir initialement "décidé de faire une parodie de fond de ces narrations totalement primaires ${ }^{6}$ afin de faire passer aux gens le goût de cette 
littérature d'abrutissement à bulles, spécialement conçue pour les analphabètes $\gg^{7}$. Première cible de son intention parodique, ces surhommes en justaucorps bariolés et moulant des musculatures spectaculaires - ces Flash Gordon (Chester Gould/1931), Superman (Jerry Siegel-Joe Shuster/1938), et autres Batmann (Bob Kane-Bill Finger/ 1939) pour n'en citer que les plus connus, purs produits d'un « marché de super-héros de plus en plus infantilisant ${ }^{8}$ " qui étaient, depuis les années trente, inlassablement occupés à sauver le monde'. Des personnages en effet doublement irritants pour un créateur allemand dessinant dans un pays qui avait très récemment connu (et " exporté ») l'un des pires sauveurs de l'histoire de l'humanité... Schmidt procède alors selon deux impératifs : la prise de distance par rapport au modèle américain, et, surtout, la mise en cause du principe même du surhomme/sauveur. Cette double charge parodique (que les lecteurs ne perçoivent pas toujours) sous-tend l'ensemble de la BD. Elle n'a pas empêché la popularité de Knatterton, le public ayant immédiatement plébiscité ce détective pas comme les autres. Une multitude de produits dérivés créés dès son apparition en témoigne par ailleurs : poupées, masques, jeux de cartes, alcool, et même une variété d'oranges porteront son nom ${ }^{10}$. Ses aventures paraissent sous forme de recueil dès 1952, et seront reprises plus tard en format série et dessin animé ${ }^{11}$. Un premier film sort en $1959^{12}$. La plus récente adaptation cinématographique - un flop - date de $2002^{13}$. En 2007 paraît avec plus de succès une version audio sur CD.

\section{Les passionnantes aventures du célèbre super- détective ${ }^{14}$}

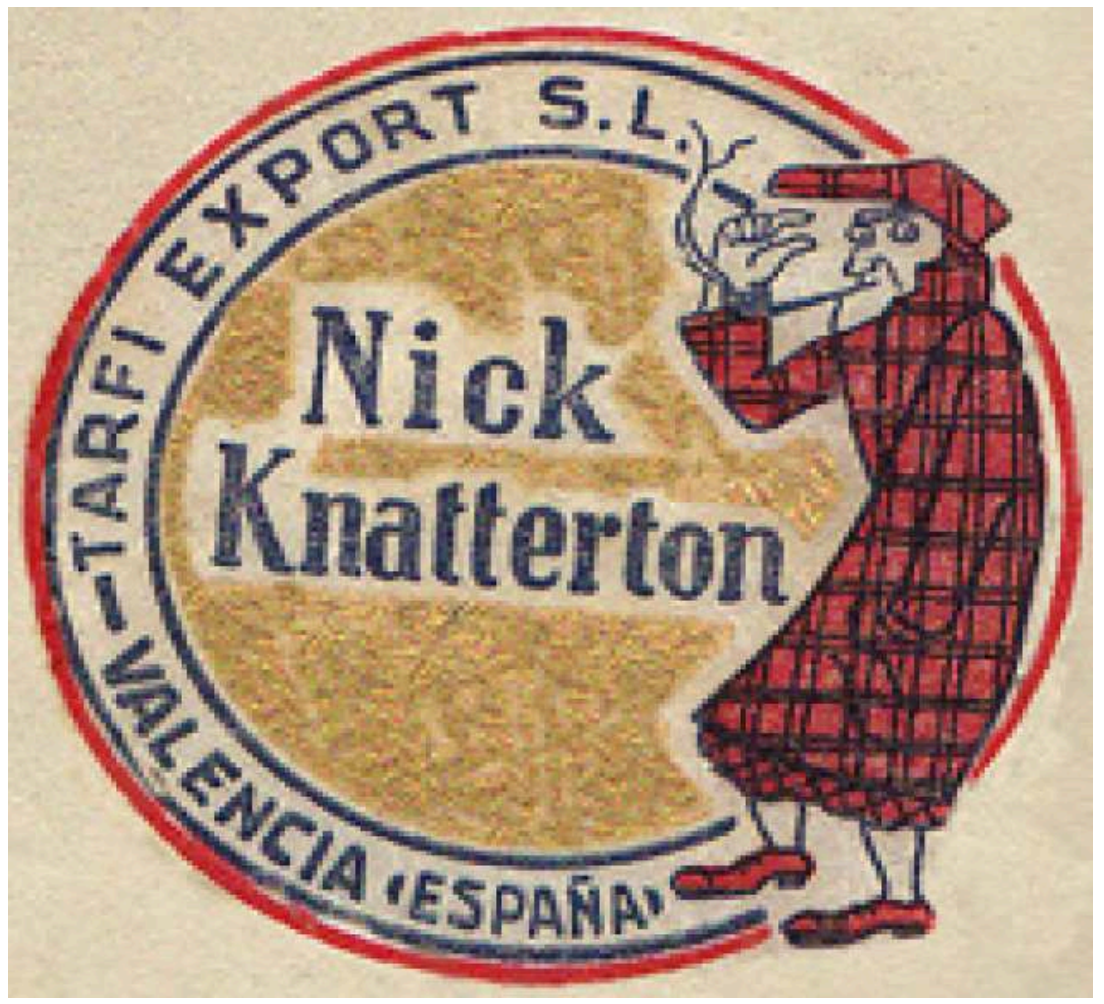

Emballage d'orange

Dix-huit aventures ${ }^{15}$ paraissent ainsi à partir du 3 décembre 1950. La série avait failli s'arrêter avec le mariage de Knatterton avec la blonde Linda Knips, photographe et 
orpheline (et accessoirement héritière d'un richissime oncle d'Amérique) qu'il rencontre dans L'héritage dans la cravate (Die Erbschaft in der Krawatte). Mais Schmidt doit céder à la pression de ses lecteurs ${ }^{16}$ et poursuivre ses "comédies urbaines ${ }^{17}$ au scénario quasi immuable: un millionnaire/un génial inventeur/une demoiselle en détresse demande au super-détective de le/la protéger ou de mettre en échec les malfaiteurs qui menacent de le/la dépouiller - si ce n'est pas déjà fait. S'ensuit une aventure à rebondissements opposant le Bien et le Mal (avec des majuscules) qui finit inévitablement avec le triomphe du Bien, c'est-à-dire de l'ordre établi - ou au moins de sa caricature, car chez Schmidt, peu de vestes sont blanches (et souvent plutôt retournées), les fortunes sont pour la plupart douteuses, les inventions le plus souvent susceptibles d'anéantir l'humanité et les innocences rarement aussi pures qu'elles ne le paraissent... La mise en scène graphique et textuelle des aventures (dessins et textes de Schmidt) suit également un protocole fixe : un titre plus ou moins absurde (p.ex. La lettre de menace dans le pyjama/Der Drohbrief im Pyjama) précède un ensemble de cinq à six vignettes destinées à introduire les principaux personnages ${ }^{18}$, accessoires ${ }^{19}$ ou encore principes ${ }^{20}$ des différentes histoires. Celles-ci comprennent entre 10 épisodes pour les plus courtes et trente pour les plus longues. L'hebdomadaire Quick publiedeux épisodes d'une aventure par numéro, chacun sous-titré et comprenant deux bandeaux («strips ») par épisode. La publication complète d'une aventure oscille ainsi entre onze et trente-huit semaines. Un épisode comprend entre cinq et neuf vignettes de taille variable, parfois si chargées que le regard du lecteur a du mal à se fixer malgré le trait très précis de cette $\mathrm{BD}$ en noir et blanc ${ }^{21}$. L'occasionnelle non-linéarité du récit apparaitt comme une manière supplémentaire de parodier un genre de narration a priori méprisé. La dynamique du récit provient des ellipses, mais paradoxalement aussi des innombrables inserts (commentaires et explications ${ }^{22}$, plans de ville et de déplacements, graphiques, schémas ${ }^{23}$ etc.) dont Schmidt use et abuse.

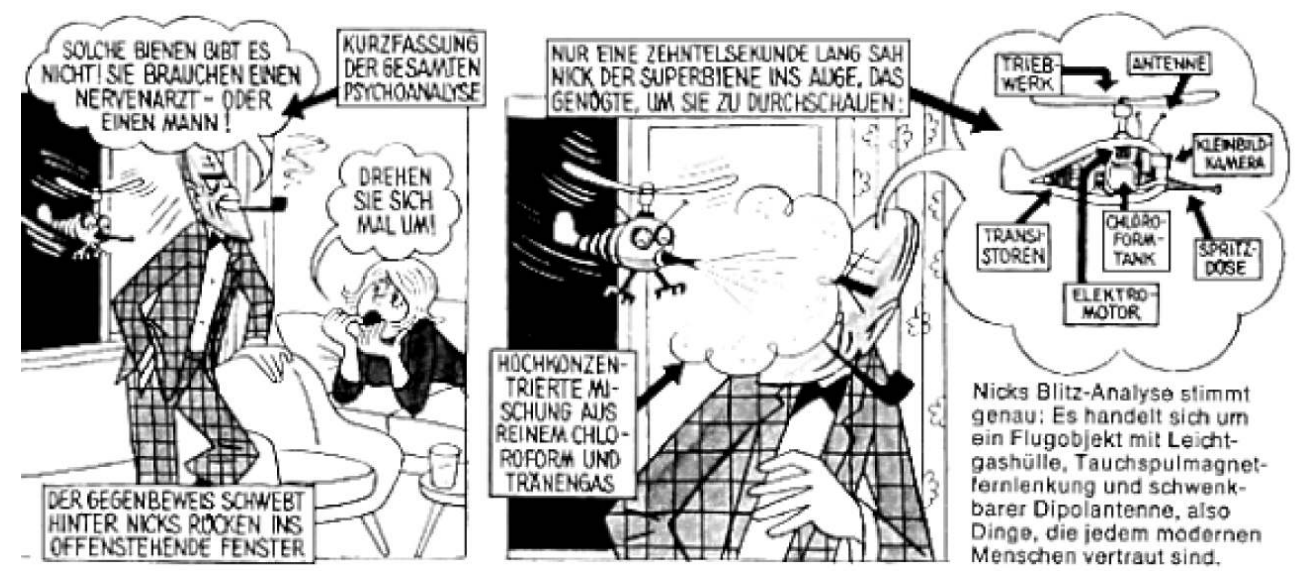

Coupe de la super-abeille espionne dans Le mystère de la super-abeille, (CA.Riedhammer

5 Ces textes se distinguent des bulles elles-mêmes par leur format, des cartouches carrés qui font le plus souvent éclater le cadre des vignettes qu'elles séparent et/ou relient. Ce sont des temps d'arrêt sur image où Schmidt, qui n'est pas créateur à prétendre vouloir disparaître derrière son personnage, s'adresse directement au lecteur. 


\section{Le monde de Knatterton}

\section{Knatterton}

«Il mesurait un peu plus d'un mètre quatre-vingts, mais il était si excessivement mince qu'il paraissait beaucoup plus grand. Ses yeux étaient vifs et perçants [...]. Son nez aquilin et fin donnait à sa physionomie un air attentif et décidé. La forme carré et proéminente de son menton indiquait aussi l'homme volontaire. [...] $»^{24}$.

6 La description que Watson fait de son futur colocataire Sherlock Holmes avec qui il partagera bientôt la célèbre adresse du $221 \mathrm{~b}$ Baker Street pourrait être celle de Knatterton... Les deux fument d'ailleurs la pipe, et dans Le million est dans le lac (Die Million im Eimer), on aperçoit Knatterton jouant du violon, instrument que Holmes, nul ne l'ignore, pratique dans les moments de grande tension intellectuelle. Le code vestimentaire (costume à pantalon knickerbocker à carreaux écossais, casquette à carreaux assortie ${ }^{25} .$. ) de Knatterton les rapproche également, même si Doyle avait présenté son détective plutôt comme une sorte de dandy de la criminologie portant parfaitement redingote et tenue de soirée. Mais dans l'imaginaire de ses fans, le style gentleman-farmer avec son deerstalker ${ }^{26}$ était devenu la marque de fabrique de Holmes à partir des premières illustrations et adaptations cinématographiques de ses aventures. De même, Holmes n'a, foi de holmsélogues, jamais prononcé la phrase « élémentaire, mon cher Watson " qu'on a l'habitude de lui attribuer ${ }^{27}$. Knatterton en revanche répète effectivement ad libitum son fameux «Kombiniere ! " (j'en déduis que...) quand les morceaux du puzzle finissent par se mettre en place. Tel l'eurêka d'Archimède, cette exclamation annonce la résolution partielle ou globale d'une énigme, et ponctue l'aboutissement de sa méthode d'investigation (observation, analyse des indices et synthèse logique/explication) calquée sur la "science de la déduction » holmésienne ${ }^{28}$ qui n'est cependant pas, tout comme pour son modèle, à l'abri de certains ratés ${ }^{29}$. Ajoutons que l'une des aventures de Knatterton, La tête dans la Tamise (Ein Kopf fiel in die Themse) ${ }^{30}$, a comme toile de fond Londres, hommage probable à son illustre prédécesseur.

7 Nick Carter et Nat Pinkerton seraient à l'origine du nom de Nick Knatterton. Et c'est sans doute aux détectives américains des années trente et quarante, héros des romans noirs de Dashiell Hammet, John Huston, Mickey Spillane etc. que Knatterton doit certains traits de caractère comme cette nonchalance parfois proche du désabusement d'un Sam Spade... Plus original, le recours massif à des gadgets techniques, souvent comme éléments centraux de la série. Une innovation en parfaite cohérence avec la très forte foi des années cinquante en un progrès que l'on veut voir comme porteur de salut. Dès 1950, Schmidt anticipe ainsi, en le parodiant déjà, un personnage qui ne verra le jour qu'en 1953: James Bond ${ }^{31}$. Ce superman de la Guerre froide - né, selon la biographie imaginaire de John Pearson, à Wattenscheid près d'Essen et par conséquent parfaitement bilingue allemand - mènera à bien ses croisades contre le Mal avec des accessoires technologiques parfois assez délirants dérivés des recherches au service de la conquête spatiale, éminent enjeu de l'après-guerre. Les gadgets de Knatterton sont bien entendu plus modestes voire - intention parodique oblige - plus farfelus. Mais il a quand même recours à des clés et des tournevis cachés dans les talons de ses chaussures, à des pointes américaines et des parachutes dissimulés dans de fausses barbes, des voitures truquées, des microfilms dans des postiches voire dans une fausse dent $^{32}$, des émetteurs dans des pièces de monnaie etc. Même le parapluie empoisonné, 
accessoire indispensable de l'affrontement entre les blocs est et ouest, n'est pas oublié... Les chaussures de Knatterton peuvent opportunément se transformer en rollers, la déjà mentionnée abeille-espionne lui sert d'indic, des "airbags " à auto-déclenchement dissimulés dans ses fonds de pantalon atténuent ses chutes... Impayable, ce faux crâne où se fichent les balles qui lui sont destinées (et qui donne son titre à l'une des premières aventures: La balle dans le crâne artificiel (Der Schuss in den künstlichen Hinterkopf). La parodie transpire aussi dans la manière de traiter la question des déguisements. Conan Doyle avait décrit Holmes comme un génie en la matière. Une fois déguisé, même Watson ne le reconnaît plus. Les super-détectives américains ou Bond méprisent l'idée même du travestissement. Knatterton pour sa part est présenté comme le maître absolu du déguisement auquel il a fréquemment recours, mais ces derniers sont si transparents que personne ne peut sérieusement imaginer qu'il puisse tromper ses adversaires ne serait-ce qu'une seconde avec des accessoires soulignant plus qu'ils ne la modifient une silhouette reconnaissable entre toutes. Contradiction que le trait de Schmidt accentue avec une délectation évidente...

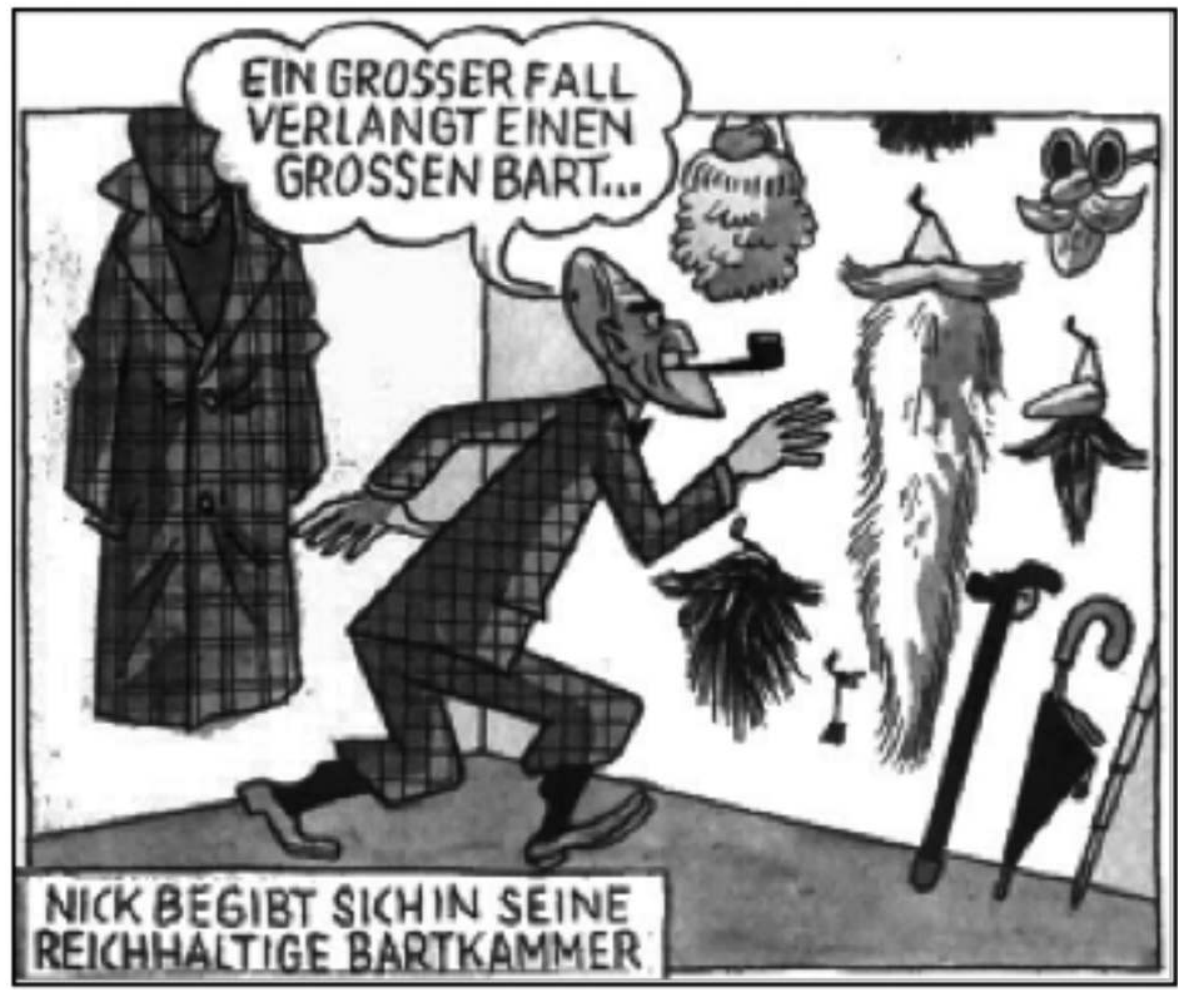

Der Schuss in den künstlichen Hinterkopf, ( A A Riedhammer

Délectation avec laquelle il compose aussi la biographie imaginaire de son détective ${ }^{33}$ :

Son « vrai » nom : Nikolaus Freiherr von Knatter.

Son lieu de naissance : le château familial des von Knatter, à Kyritz sur Knatter

Sa mère: Corinna Freifrau von Knatter, née Freiin Peng ${ }^{34}$ zu Bimsburg, grande amatrice de littérature policière (passion transmise à son fils).

Son père: Botho Freiherr von Knatter, lieutenant-colonel à la retraite, et «maître du calembour » (un don dont son rejeton a indéniablement hérités5).

Montrant une curiosité et des qualités exceptionnelles de raisonnement ainsi que de déduction dès son plus jeune âge, Nikolaus von Knatter décide, au grand dam de ses parents, de devenir détective privé. Un conseil de famille, soucieux de préserver la 
réputation de la lignée, l'oblige alors à prendre un "pseudonyme » : Nick Knatterton est né...

\section{Knatterton et les femmes}

10 Knatterton est constamment entouré de créatures pulpeuses à souhait, détail que Schmidt affirme lui avoir été soufflé par la rédaction QUICK qui lui aurait conseillé de pourvoir les personnages féminins - honnêtes ou fatals - de généreux appas. « Dessinez des poitrines voluptueuses et des derrières appétissants, et ce sera un succès $»^{36}$ - telle était la consigne. Il crée donc des silhouettes à la féminité exacerbée, en parfaite adéquation avec le physique sensuel et opulent de certaines actrices des années cinquante, Marilyn Monroe en tête qu'il immortalise d'ailleurs dans l'une des aventures sous les traits de la starlette Marylinerl Mees.

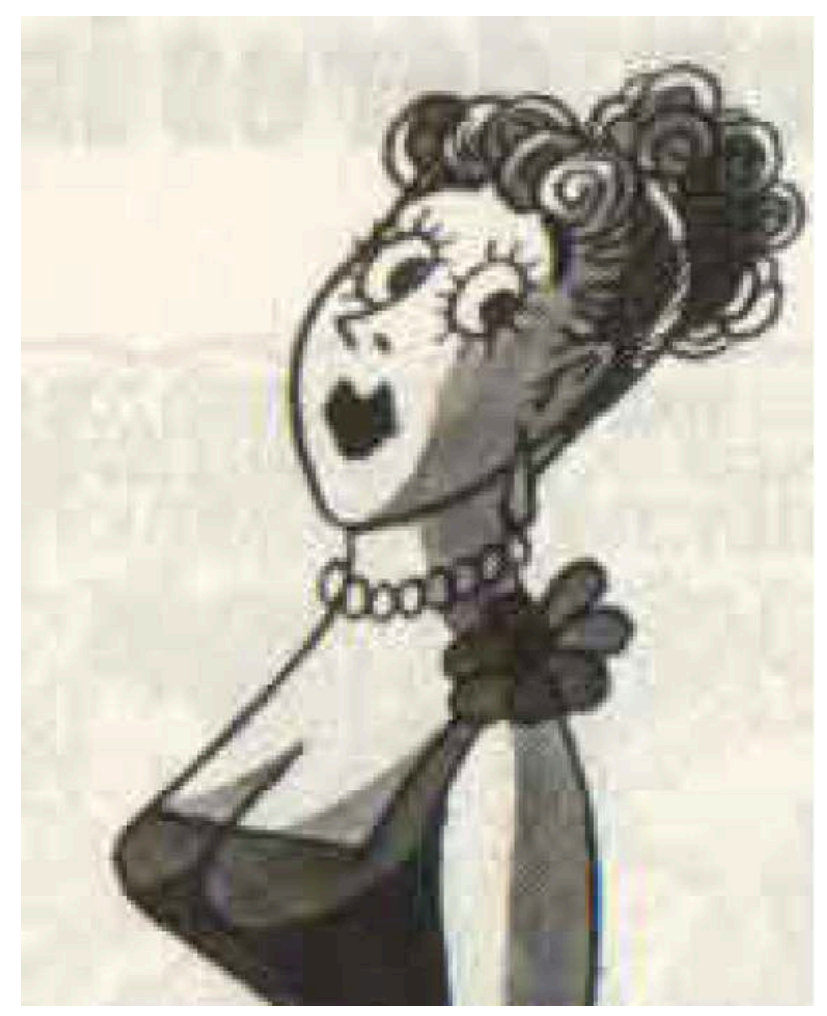

Linda Knips 


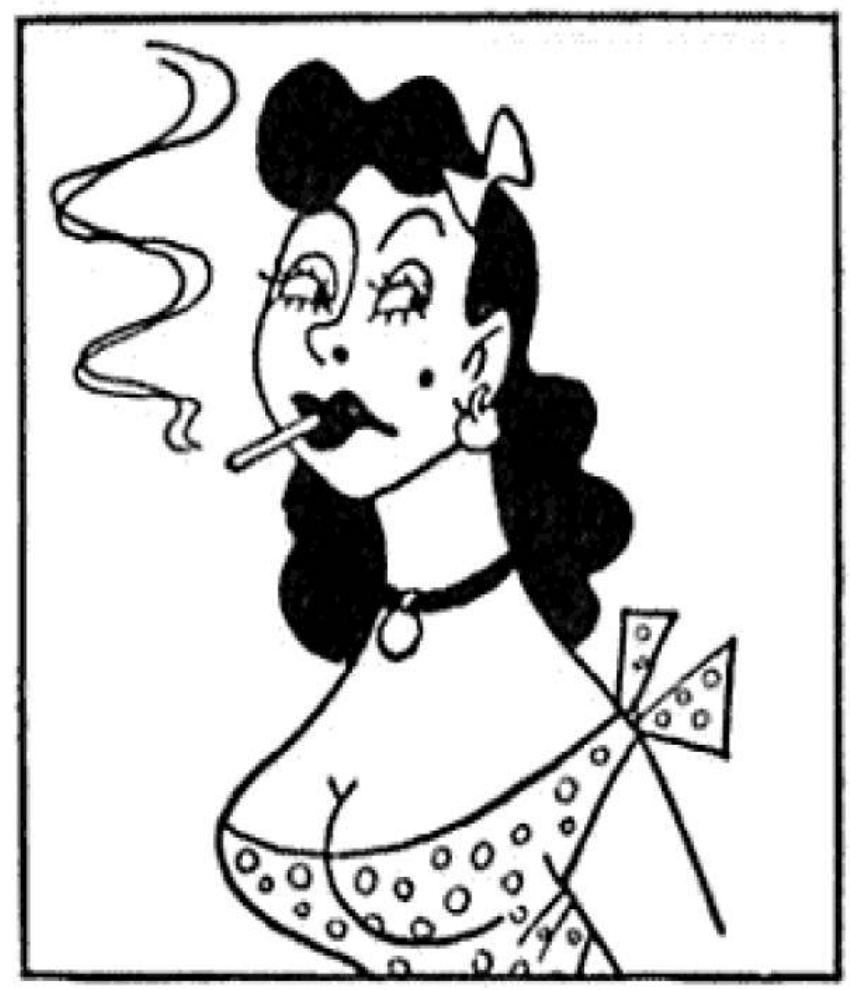

Virginia Peng

Selon un principe classique de la $\mathrm{BD}$, Schmidt dote ses personnages féminins - et masculins d'ailleurs - de noms parlants annonçant leur rôle ou fonction dans l'aventure. On ne résiste pas au plaisir de citer la fiancée de gangster Virginia Peng, Priscilla Cornflake (une jeune fermière du Middlewest), l'hôtesse de l'air Tilly Clipper, la « fleur du trottoir » Olga Kokottkaja, la secrétaire Tilly Tipp, la danseuse nue Dolly Doll, la vénéneuse Mi-Tse Meyer (fruit des amours entre une cavalière rhénane et un jongleur chinois qui ne supporte pas, mais vraiment pas du tout qu'on l'appelle «Mieze »), Lori Zontal (initialement prénommée Hori) ou encore la richissime veuve argentine Carmen Pesetas. À première vue nuancés, ces personnages ne reproduisent en fin de compte que la catégorisation traditionnelle de «l'éternel féminin »: la vamp ou la madone, qui ont en commun leur volonté de séduire Knatterton pour le meilleur (amoureuses certes, mais visant fatalement le mariage) ou le pire (elles servent d'appât à de potentiels assassins). Mais pas plus qu'à Holmes (dont on connait la passion sans doute jamais assouvie pour Irène Adler) ou Bond (celle qu'il épouse dans Au Service Secret de sa Majesté est tuée juste après les noces), le mariage ne sied à Knatterton. Ayant dû promettre à sa femme, la déjà mentionnée Linda Knips, de ne plus se mettre en danger, il s'ennuie ferme dans son luxueux foyer conjugal et sombre dans la dépression. En épouse compréhensive, Linda finit par lui « organiser » une aventure (Véridium 275, où apparaît l'autre grand classique de la Guerre froide, le sérum de vérité). Knatterton reprend immédiatement du service et goût à la vie, résout le problème qui avait fini par se transformer en véritable cas - et pardonne sa ruse à Linda. Celle-ci continuera d'être occasionnellement présente dans les épisodes suivants. La tentative de Schmidt d'adjoindre à Knatterton un Watson miniature de quatorze ans nommé Toni Knatter ${ }^{37}$ faisant accessoirement fonction de fils de substitution échoue en revanche. Les héros n'ont pas de famille : même marié, le grand détective est forcément un grand solitaire. Et un dur: une rare vignette (toujours dans Véridium 275) nous le montre les yeux 
humides quand il recueille la confession d'une belle aux formes provocantes qui s'est opportunément évanouie dans ses bras (pas trop) musclés... Au lecteur d'interpréter l'origine de son inhabituelle émotion.

L'univers de Schmidt est donc tout à fait conservateur, même si nombre de femmes travaillent (au cours des années cinquante, elles arrivent massivement sur le marché du travail). Leurs emplois, inévitablement subalternes, sont parfois classiques (la secrétaire Tilly Tipp est la réactivation directe de la dactylo des années vingt ${ }^{38}$ ), parfois plus glamour (Tilly Clipper est hôtesse de l'air, métier dont rêvent les jeunes filles à une époque où le transport aérien est encore largement réservé aux élites). Toutes attendent en réalité le prince charmant et le mariage, leur " vrai » statut social. Ecueil que Knatterton le justicier réussit à repousser longtemps en affirmant être marié avec son travail...

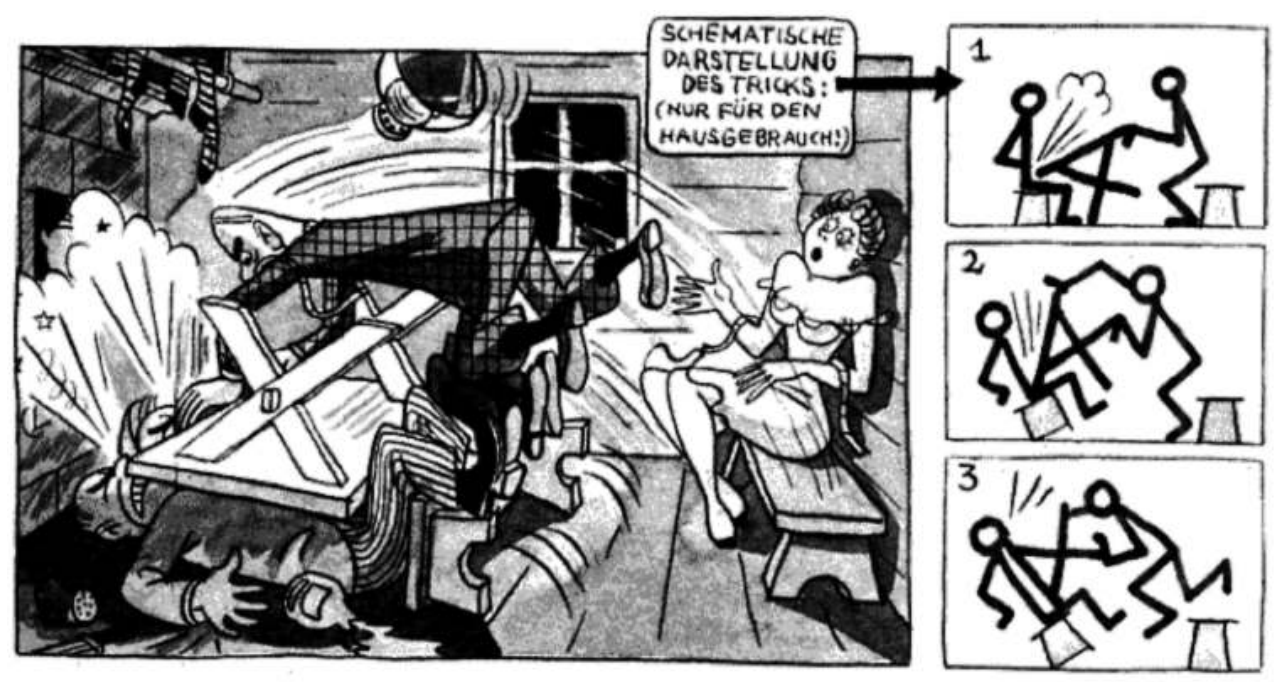

Der Schuss in den künstlichen Hinterkopf, (C) A.Riedhammer

Dans un tel environnement, la photographe Linda Knips pourrait, à première vue, presque faire figure de femme émancipée ${ }^{39}$. Mais elle aussi n'aspire au fond qu'à fonder un foyer pour y enfermer son homme. Elle fait partie de ces madones de préférence blondes, l'opposé des vamps brunes et lourdement maquillées (cf. Virginia Peng) issues de ces mondes interlopes où se préparent les mauvais coups, et qui sont en fin de compte plus «dangereuses » que ces dernières. Quelle que soit cependant leur couleur de cheveux, madones et vamps usent et abusent également de stratagèmes «typiquement féminins" (vraies ou fausses larmes, évanouissements opportuns, vêtements plus ou moins transparents) qui sont autant d'appels classiques au réflexe de protection masculin. Et cela fonctionne particulièrement bien avec un Knatterton toujours prêt à tomber dans TOUS les panneaux. Ce super-héros doté d'une intelligence bien au-dessus de la moyenne s'avère un indécrottable naïf en ce qui concerne les femmes. Le détective dont Schmidt souligne inlassablement - et ironiquement ! - la supériorité intellectuelle se trouve ainsi systématiquement en position d'infériorité devant les ruses féminines. Sa tenue vestimentaire est peut-être l'indice malicieux de ce talon d'Achille: le knickerbocker n'est après tout qu'une variante de la culotte courte, vêtement traditionnel des adolescents des années vingt et trente... 


\section{Protagonistes et antagonistes}

Tout comme les personnages féminins, les personnages masculins correspondent à des stéréotypes de l'époque. De richissimes commanditaires, des capitaines d'industrie, des magnats de la finance etc. Des self-made-men à l'exemple de Karl Murx né Marx dont le nom traduit parfaitement la «mutation », le multimillionnaire Lucius X. Nylon (alias DuPont de Nemours...) ou encore Egon Knicker qui règne sur un nouvel empire de grands magasins en constante extension. Sans commentaire Bruno Bims, "grand patriote » et "fournisseur de l'armée depuis quatre générations »- et bien entendu impuni dans une Allemagne qui avait voté dès la fin des années quarante l'amnistie de ses élites économiques, sous l'impulsion du chancelier Adenauer qui estimait qu'elles devaient pouvoir participer à la reconstruction du pays. Quelques derniers rejetons de maisons régnantes (le nabab indien Nawarthmal von Lextropur) ou de l'aristocratie (le très british Lord Spleen). Ils ont en commun d'être obsédés par leurs fortunes plutôt mal que bien acquises, au mieux héritées, mais très rarement obtenues grâce à un travail honnête. Tous sont constamment menacés par la pègre (Bruno Bluff, Max Klaut, Macco Maffiano...), ou par des collaborateurs prêts à les trahir (l'indélicat comptable Sigmund Saldo).

15 À nouveau deux mondes qui se traduisent par deux esthétiques opposées. Aux premiers les intérieurs cossus conçus sur le modèle américain (villas de style bungalow aux baies vitrées, mobilier contemporain, tableaux et sculptures modernes ${ }^{40}$, équipements technologiques dernier cri etc. ${ }^{41}$ ), aux autres des bas-fonds pittoresques à souhait correspondant à un imaginaire parfaitement exprimé par l'un des «tubes » de la fin des années cinquante: tango criminale dans la taverne/louches les silhouettes, rouges les lanternes/soir après soir, la mèche crépite/une tension palpable remplit l'atmosphère ${ }^{42}$. Les malfaiteurs qui évoluent dans ces décors sont des gangsters d'opérette, et plus prosaïquement des représentants des classes sociales inférieures, les éternels perdants de l'Histoire - et des histoires de Knatterton.

\section{Nick Knatterton et l'Allemagne des années cinquante}

Tout au long des années cinquante, l'Allemagne se reconstruit, assoit sa stabilité politique, renoue avec une rapidité assez spectaculaire avec la prospérité matérielle, atteint le plein-emploi, démocratise l'accès aux études supérieures, favorise l'ascension sociale... C'est un État-providence qui se dessine puis s'installe, une époque charnière marquée par la fixation du processus de renouveau démocratique reposant sur la Loi fondamentale votée le 23 mai 1949. L'économie sociale de marché défendue par Ludwig Ehrhardt (ministre de l'économie de 1949-1963, chancelier de 1963-1966) porte ses fruits. Les premiers supermarchés proposent le «nouveau Persil » (das neue Persil), des Tampax (« Maintenant aussi en Allemagne!»/ Jetzt auch in Deutschland!), et du Coca-Cola, boisson dans le vent à laquelle la publicité - ou plutôt la réclame! - de l'époque attribue d'ailleurs des vertus quasi médicinales ${ }^{43}$. Les ménages commencent à s'équiper: les premiers lave-linge, réfrigérateurs, rasoirs électriques et aspirateurs (l'aspirateur-boule de chez Hoover!) font leur entrée dans les coquets pavillons des classes moyennes devant lesquels stationnent les Mercedes - symbole de la réussite du chef de famille - que l'on bichonne dûment le dimanche. Les trouble-fête ont beau 
mettre en garde contre une conception de plus en plus matérialiste de l'existence, l'élan est donné : le Zeitgeist avait produit la naissance de la société de consommation.

Ces «années Knatterton " avec leur foi dans le progrès et la vertu du travail, radicalement tendues vers l'avenir après les épreuves de la guerre, pas trop regardantes quant à certaines responsabilités pourtant avérées, ne résisteront cependant pas aux coups qui les ébranleront à partir des années soixante: le mouvement hippie, l'essor du féminisme, la contestation estudiantine (et la mort de Rudi Dutschke), la mobilisation contre le nucléaire (Tchernobyl sera pour 1986), la RAF et Baader-Meinhof, mais aussi les premiers chocs pétroliers, les premières manifestations pour la protection de l'environnement et des ressources naturelles etc. sonneront le glas d'une ère soudainement démasquée comme une fausse idylle. Pour la génération des parents, ces années cinquante représentaient une période d'espoir après la tempête. Pour les enfants du baby-boom, elles avaient produit une société à la morale insupportablement hypocrite, petite-bourgeoise et coincée qu'il convenait de faire exploser. Knatterton est alors tout simplement dépassé. Tout comme la table basse en forme de rognon («Nierentisch »), sa nouvelle ringardise le relègue au magasin des antiquités. Le 4 novembre 1985, le tout jeune (et premier) ministre de l'environnement et de l'énergie du Land de Hesse exprime encore ce rejet en déclarant dans une interview accordée au Spiegel dont il fait la une (titre: "Joschka Fischer, terreur de l'industrie » / Industrieschreck Joschka Fischer) combien il est heureux de quitter la ville de Bonn et "cette ambiance à la Nick Knatterton, ce style des années cinquante " ${ }^{44}$. Le verdict semble sans appel. Mais comme le design des années cinquante, Knatterton n'a jamais disparu de la mémoire culturelle collective de l'Allemagne, et il a fait son come-back.

Et ce n'est pas à la seule nostalgie ou à une éphémère tendance esthétique que la série de Schmidt doit ce regain d'intérêt qui se confirme aujourd'hui. On a progressivement découvert la $\mathrm{BD}$ non seulement comme un art à part entière, mais aussi comme une source documentaire potentiellement intéressante et souvent pertinente pour les époques respectives de leur création ${ }^{45}$. Dans le cas de Knatterton, ce sont indéniablement les motifs de la Guerre froide avec ses gadgets devenus cultes (ah, la cloche à fromage en plexi-glass pour conférences top secrètes ${ }^{46}$ !), et du passé du pays à travers l'accumulation de tous ces personnages aux biographies et carrières douteuses ${ }^{47}$ qui retiennent l'attention... C'est un univers paranoïaque où un banal bidon de lait peut se transformer en arme fatale, un pays truqué dont la réussite démocratique et économique s'avère construite sur des compromis et compromissions peu acceptables. 


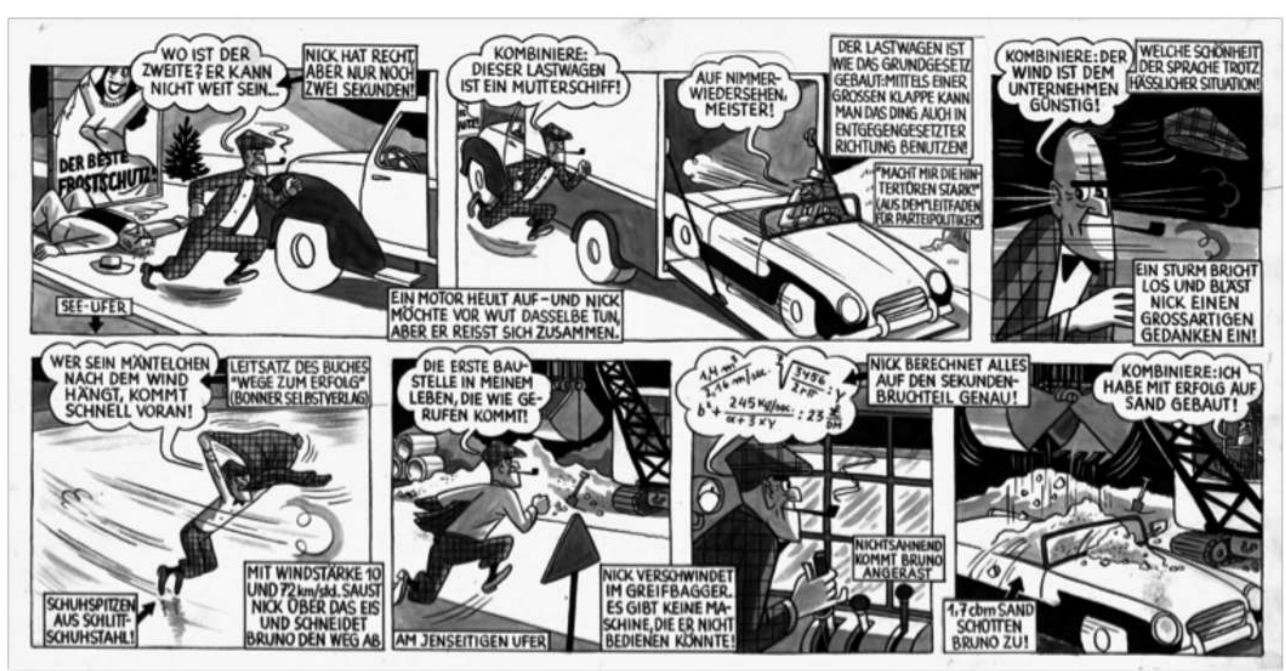

275, (C) A.Riedhammer

Dans La Valise aux diamants indienne, L'Inde, double de l'Allemagne, est une « dictature démocratique " ou tout doit être considéré à travers les "lunettes des partis " (Parteibrille). Dans La tête dans la Tamise, la capitale anglaise nage dans le brouillard, tout comme le gouvernement allemand. Le monogramme sur le mouchoir de Sigmund Saldo ressemble à s'y méprendre à une croix gammée (Le million est dans le lac). Dans Le savon à barbe comme arme secrète (Die Rasierseifen-Geheimwaffe), Schmidt se livre à des variations sur le terme et le thème du suiveur (Mitläufer). Dans Le secret derrière le hublot, un coucher de soleil éclaire les montagnes de l'arrière-pays dans un beau dégradé de « brun nazi au noir CDU » (Übergänge von NS-Braun bis zu CDU-Schwarz)... Passé et présent se rejoignent, et rarement pour le meilleur.

20 À la parodie du genre et la critique de société s'ajoute l'analyse en temps quasiment réel d'un quotidien allemand de plus en plus confortable, mais aussi de plus en plus aliénant et incompréhensible: consumérisme effréné, impôts arbitraires, argent fétiche, accointances et décisions politiques aberrantes, faits divers spectaculairement sordides et valeurs perdues... Schmidt colle à l'actualité : dans certains numéros de Quick, l'épisode de Knatterton pouvait traiter du même sujet que l'article de fond. Cela permettait d'épingler en temps réel certains événements culturels, politiques, sociétaux. Tout au long de sa production, Schmidt avait su garder sa capacité d'ironie à tiroirs qui caractérise de manière si réjouissante l'ensemble de la série. Elle pouvait d'ailleurs s'étendre à son propre métier de BDiste: dans Une porte perd sa serrure (Ein Schloss fällt aus der Tür ${ }^{48}$ ), l'entrepreneur véreux Heitz est aussi membre d'une ligue de vertu militant pour la protection de la jeunesse, et réclamant à cor et à cri l'interdiction de la bande dessinée... 


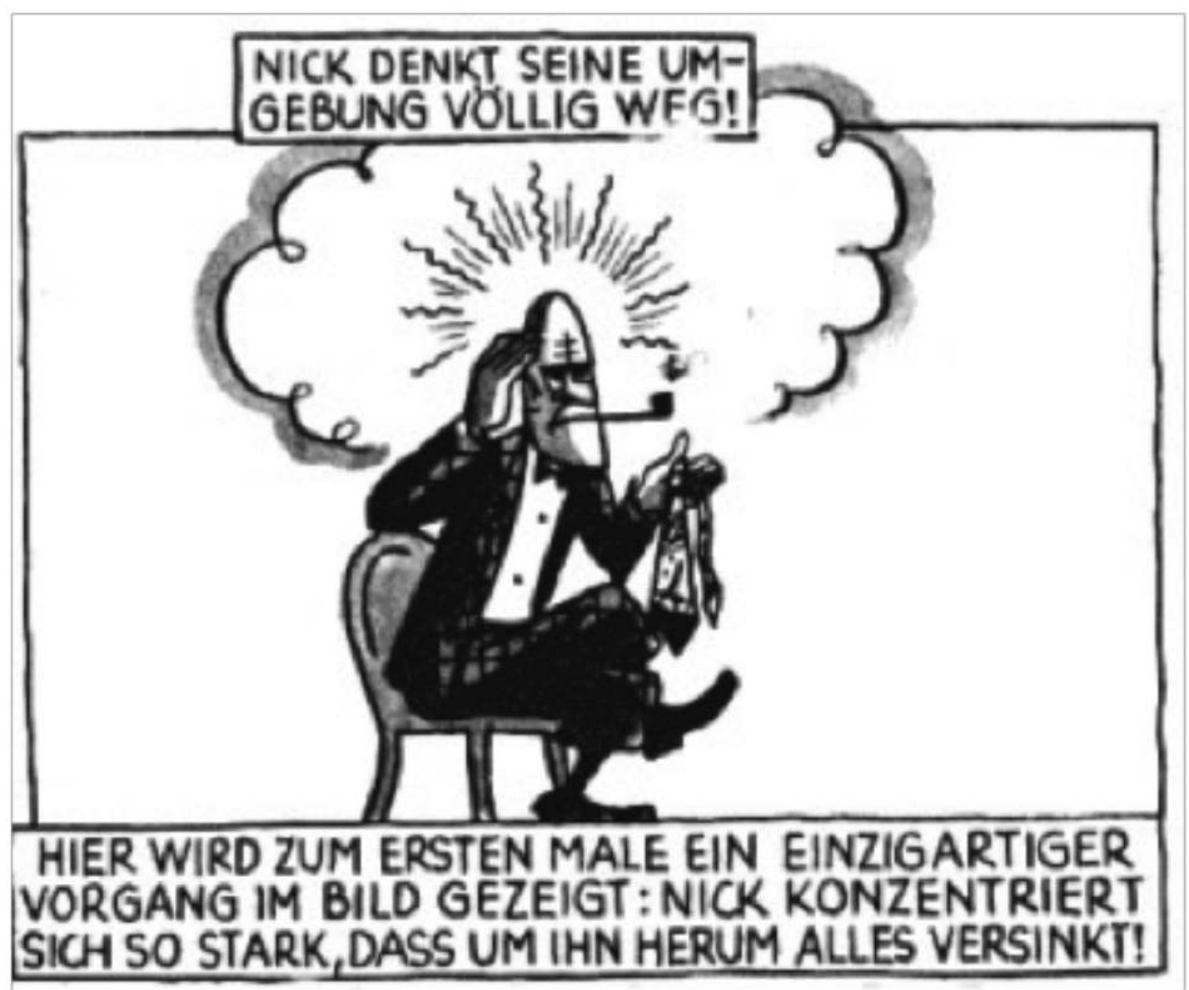

Die Erbschaft in der Krawatte, ( A. Riedhammer

\section{NOTES}

1. «Der erste wirkliche Klassiker des deutschen Comics ». Voir à ce sujet Bernd Dolle-Weinkauff, Comics - Geschichte einer populären Literaturform in Deutschland seit 1945, Weinheim und Basel, Beltz, 1990.

2. Tout en y ayant recours pour sa propre propagande.

3. Méfiance loin d'avoir disparue de nos jours comme l'affirme Alain Chante: «[...] des opinions désuètes sur les dangers que la BD fait courir à la lecture, sur le simplisme de ses histoires ou sur l'infantilisme de ses lecteurs, persistent encore. » In : 99 réponses sur la bande dessinée, CNDP/CRDP, Montpellier, 2000, préface.

4. Lire à ce sujet le récent ouvrage retraçant la véritable chasse aux sorcières contre la $\mathrm{BD}$ à laquelle s'était livrée l'Amérique des années cinquante: David Hajdu, The Ten-Cent Plague. The Great Comic-Book Scare and How it Changed America (La peste à dix sous. La grande peur des BD et comment elle a changé l'Amérique), Farrar, Strauss \& Giroux, 2008.

5. «De l'opium dans la chambre d'enfants» (Opium in der Kinderstube), cf. Andreas C. Knigge, «Zeichen-Welten. Der Kosmos des Comics», in: Heinz Ludwig Arnold Hrsg., Text+Kritik V/09 (Comics, Mangas, Graphic Novels), p. 20. En Allemagne, les détracteurs de BD n'hésitent pas à les brûler sur la place publique...

Traduction française des citations par l'auteure. 
6. Au sujet de la parodie dans le domaine de la BD l'article d'Alain Chante, «La parodie en sept leçons ", in : Les Cahiers de la Bande Dessinée, bimestriel, n61/1985, p. 72-75.

7. « Ich nahm mir vor, diese primitivste aller Erzählformen so gründlich zu parodieren, dass den Leuten alle Lust an der blasenreichen, auf Analphabeten zugeschnittene Stumpfsinnliteratur verging." Cf. l'introduction signée Manfred Schmidt in: Nick Knatterton. Alle aufregenden Abenteuer des berühmten Meisterdetektivs. Oldenburg, Lappan-Verlag, 1983, p. 5. Les aventures citées à la suite renvoient à cette édition.

Je remercie $\mathrm{M}^{\mathrm{me}}$ Annette Riedhammer pour l'autorisation à reproduire certains strips et images dans ce travail.

8. «Der immer infantilere Superheldenmarkt», cf. Wolfram Knorr, «Will Eisner. Das visuelle Rauschgift des Bilderromans ", in : Text+Kritik V/09, op. cit., p. 81.

9. Lire ou relire à ce sujet Umberto Eco [1978], «Le mythe de Superman », in : De Superman au surhomme, traduction française de Myriem Bouzaher, Grasset, 1993, p. 131-170.

10. Schmidt [1983], 4-7.

11. C'est Schmidt en personne qui est, en 1978, à l'origine du dessin animé conçu pour l'ARD (un pilote et 14 épisodes rebrassant les histoires originales) et qui la commente en voix off. Disponible en DVD et sur internet.

12. Die Abenteuer des Nick Knatterton - Der Raub der Gloria Nylon, mise en scène de Hans Quest. Le rôle principal était tenu par Karl Lieffen.

13. Metteur en scène : Niki List, musique : Reinhold Heil.

14. Je reprends ici avec une légère variation le sous-titre de l'album paru chez Lappan.

15. Le volume de 1983 (cf. note 7) n'en réunissait que 16. Inexactitude rectifiée par les éditions de 1998 et 2007 (également chez Lappan).

16. Anecdote qui n'est pas sans rappeler Conan Doyle obligé de ressusciter Sherlock Holmes après avoir orchestré sa disparition dans les chutes de Reichenbach.

17. «Urbane Komödie », cf. Andreas C. Knigge, «Zeichen-Welten. Der Kosmos des Comics », in : Text+Kritik V/09, p. 12. La ville est le principal décor des aventures de Knatterton.

18. Dont le «grand inconnu » (Der grosse Unbekannte) représenté par un point d'interrogation dans la vignette de présentation de l'aventure Vendredi soir à neuf heures (Freitag abend um neun)

19. La cravate/carte au trésor dans L'héritage dans la cravate, la valise/coffre-fort dans La valise aux diamants indienne (Der indische Diamantenkoffer) etc.

20. Exemple: «La vérité jouera un rôle principal. Le buste que vous apercevez ici, créé par un artiste moderne, la montre les seins voilés, car la vérité nue n'est que rarement belle. Elle a le cœur sur la langue et brandit son flambeau. Comme on peut s'y attendre après plusieurs millénaires d'expérience, ses cheveux se dressent sur sa tête." (Die Wahrheit wird eine Hauptrolle spielen. Die hier gezeigte, von einem modernen Künstler geschaffene Büste trägt einen verhüllten Busen, denn die nackte Wahrheit ist nur selten schön. Sie trägt ihr Herz auf der Zunge und die Fackel hoch. Wie nach jahrtausende alten Erfahrungen nicht anders zu erwarten ist, stehen ihr die Haare zu Berge. Schmidt [1983], Veridium 275, p. 155).

21. Choix esthétique pour une BD destinée aux adultes mais sans doute aussi parti pris d'une représentation caricaturale du monde à l'époque de la Guerre froide.

22. Toujours dans Veridium 275, l'un de ces textes est même à lire à l'envers. Cf. Schmidt [1983], p. 156.

23. Dont la visée est parfois "didactique »: exemple le schéma du circuit imprimé de l'émetteur caché dans une pièce de 5 Mark dans Le secret de la super-abeille qui est précédé du commentaire "pour les lecteurs qui voudraient construire un tel dispositif» (für Leser, die sowas bauen wollen), Schmidt [1983], p. 106.

24. Cf. Arthur Conan Doyle [1887], Étude en rouge (A study in scarlet), traduction de Pierre Baillargeon, préface de Germaine Beaumont, Livre de poche, 1979, p. 33/34. 
25. Même le pyjama de Knatterton est taillé dans un tissu à carreaux...

26. Chapeau de chasse porté à la campagne.

27. La réplique adressée à Watson «Peuh, L'enfance de l'art!»/ it was the simplicity itself (Étude en rouge, p. 51) serait, d'après les spécialistes, à l'origine de l'expression.

28. Exposée par Holmes dans Étude en rouge, op.cit., p. 42.

29. À ce sujet, la tout aussi instructive qu'amusante enquête dévoilant «le vrai coupable » de Pierre Bayard, L'Affaire du chien de Baskerville, Les Éditions de Minuit, 2008.

30. Dont l'un des principaux « personnages » est... le fog londonien.

31. Première apparition dans Casino Royal (Ian Fleming).

32. Cachée, à l'insu de son propriétaire, dans la mâchoire de Camillo Tenorani, pêcheur à Capri et doté, on le devine, d'une voix magnifique.

33. Schmidt [1983], p. 5.

34. Nom que Schmidt avait donné à l'un des principaux personnages féminins, Virginia Peng.

35. Le langage de Knatterton en est presque essentiellement composé et mériterait à lui seul une étude approfondi.

36. Wenn Sie viel Busen und stramme Popos hineinzeichnen, wird es ein Erfolg., Schmidt [1983], p. 5.

37. Modèle réduit de Knatterton, il déduit (« Kombiniere ») même quand il n’y a rien à déduire...

38. Voir à ce sujet p.ex. Siegfried Kracauer [1930], Die Angestellten, Suhrkamp, Frankfurt/Main, 1971.

39. Grâce à Knatterton, elle est de plus financièrement indépendante.

40. Qui courent parfois le risque d'être confondues avec un vélo accidenté...

41. L'appartement de Knatterton correspond lui aussi à cette idée du confort moderne.

42. Kriminaltango in der Taverne: / dunkle Gestalten, rote Lanterne. / Abend für Abend lodert die Lunte / sprühende Spannung liegt in der Luft. Hazy Osterwald-Quartett, " Kriminal-Tango » (Text: Kurt Feltz, Musik: Piero Trombetta), 1959. Titre français «Le tango des jonquilles». La chanson sera reprise par Nina Hagen et, en 1984, par le groupe «Die toten Hosen ». Chanson entre autres inspirée par "Hernando's Hideaway", titre-phare de la comédie musicale "The pajama Game » de Richard Adler et Jerry Ross (1954). Son interprète la plus connue est Ella Fitzgerald.

43. «Pur, sain, et bon pour tous !» (Rein, gesund und immer bekömmlich!). Source : Rendez-vous unterm Nierentisch, documentaire de Manfred Breuersbrock (1987), «Prädikat wertvoll» à la Berlinale de la même année. En DVD chez Tacker-Film.

44. Diese Nick-Knatterton-Atmosphäre, diesen fünfziger Jahre-Stil... Interview «HessenKoalition: Wie Willy wollte», in: Spiegel-online, http://www.spiegel.de/spiegel/print/ d-13514045.html, consulté le 9.12.2009.

45. Axel Koberne et Ulrich Otto l'ont examiné sous cet angle dès la fin des années soixante-dix : «Nick Knatterton. Ein bundesdeutscher Comic-strip der 50er Jahre», in: Germanistische Mitteilungen 10/1979, Bruxelles, p.15-51. Autre exemple, le travail de Wolfgang Höhne, Technikdarstellung im Comic. Der Comic als Spiegel technischer Wünsche und Utopien der modernen Industriegesellschaft. Thèse soutenue à l'Université de Karlsruhe, 2003, introduction.

46. « Die Geheimkonferenzkäseglocke ». Cf. Le million est dans le lac.

47. La trajectoire du déjà cité Karl Murx né Marx n'en est qu'un exemple parmi d'autres : après avoir fait fortune en transformant en casseroles des casques récupérés après la capitulation, il devient le principal fournisseur de casques de la Deuxième République d'Allemagne. Commentaire de Schmidt : « ein wahres Wirtschaftswunderkind» (un enfant prodige du miracle économique). Cf. Le million est dans le lac, Schmidt [1983], p. 81.

48. Jeu de mot avec l'expression allemande « eine Tür fällt ins Schloss » (Une porte se ferme). 


\section{RÉSUMÉS}

Nick Knatterton, publié à partir de 1950 et jusqu'en 1959 dans la revue Quick, est sans doute la BD la plus emblématique d'une Allemagne de l'après-guerre qu'elle accompagne et commente à un moment charnière de son histoire. Premier véritable classique de la BD allemande, parodie réjouissante du genre et satire de la société, elle a connu un énorme succès. Aujourd'hui, elle peut être considérée comme un document particulièrement intéressant sur les années cinquante, la reconstruction et le miracle économique naissant. Dans un contexte de Guerre froide où s'affrontent les blocs est et ouest, son créateur Manfred Schmidt dessine avec humour distancié et lucidité des "comédies urbaines" (Knigge) en noir et blanc qui interrogent le passé de l'Allemagne tout comme l'État-providence émergeant et la naissance de la société de consommation.

Ab 1950 und bis 1959 erscheinen in der Revue Quick die Abenteuer des Meisterdetektivs Nick Knatterton, der wohl repräsentativste Comic der deutschen Nachkriegszeit, die er begleitet und kritisch kommentiert. Knatterton, ursprünglich als Gattungsparodie intendiert, ist zweifellos der erste große Klassiker der deutschen Comicproduktion. Die außergewöhnlich erfolgreiche Serie gilt heute zusätzlich als ein durchaus relevantes Zeitdokument zu den Themen fünfziger Jahre, Wiederaufbau und Wirtschaftswunder. Im Kontext des Kalten Krieges zeichnete KnattertonSchöpfer Manfred Schmidt in einem der Konfrontation zwischen den Blöcken entsprechenden Schwarz und Weiß seine «urbanen Komödien» (Knigge), und durchleuchtete mit scharfsichtigdistanziertem Humor Deutschlands Vergangenheit, den entstehenden Wohlfahrtsstaat und die Geburt der Konsumgesellschaft.

In 1950 and up to 1959, the magazine Quick publishes the adventures of super-detective Nick Knatterton, the most emblematic German comic strip after the end of World War II. Originally intended as a parody of the American comics and super-heroes, undoubtedly funny but in the same time a sharp commentary of the fifties, Knatterton is without any doubt the first classic in the comic production of Germany, and was a huge success. Cartoonist Manfred Schmidt depictures a world in black and white, corresponding to the confrontation of Eastern and Western bloc during the Cold War. Today, Nick Knatterton is recognized as a particularly relevant documentation on the fifties in Germany, the period of reconstruction and the economic miracle. Humor, clearsightedness and distance are the weapons used by Schmidt to analyze the past of the country, the upcoming Welfare state und the beginning of the consumer society.

\section{AUTEUR}

\section{INGEBORG RABENSTEIN-MICHEL}

Université Claude Bernard - Lyon 1 